\title{
Meta-analysis brings rigor to preclinical studies of extracellular vesicles for respiratory disease
}

\author{
Erin N. Worthington ${ }^{1}$, James S. Hagood ${ }^{2}$ \\ ${ }^{1}$ Division of Pulmonology, Department of Pediatrics, Carilion Clinic and Virginia Tech Carilion School of Medicine, Roanoke, VA, USA; ${ }^{2}$ Division \\ of Pulmonology, Department of Pediatrics, University of North Carolina at Chapel Hill, Chapel Hill, NC, USA \\ Correspondence to: James S. Hagood. Division of Pulmonology, Department of Pediatrics, University of North Carolina at Chapel Hill, 450 MacNider, \\ CB\# 7217, Chapel Hill 27599, NC, USA. Email: jhagood@unc.edu. \\ Comment on: Tieu A, Hu K, Gnyra C, et al. Mesenchymal stromal cell extracellular vesicles as therapy for acute and chronic respiratory diseases: a \\ meta-analysis. J Extracell Vesicles 2021;10:e12141.
}

Received: 23 December 2021; Accepted: 10 January 2022; Published: 31 March 2022.

doi: 10.21037/exrna-21-35

View this article at: https://dx.doi.org/10.21037/exrna-21-35

Cell-based therapeutics is a relatively young and exciting area of research, with considerable promise for wideranging clinical applications for treatment of diseased and damaged tissue (1). Mesenchymal stromal cells (MSCs) are multipotent cells which can function as progenitor cells and have the capacity to stimulate repair, growth, and survival of cells and tissues (2). While MSCs have been extensively investigated for their immunomodulatory and regenerative capacity there have been some lingering safety concerns for MSC therapy. However, a large number of studies have demonstrated that MSC-derived extracellular vesicles (EVs) are able to replicate many of the beneficial regenerative effects of MSCs (3). Interest in studying the therapeutic use of MSC-EVs in a broad range of acute and chronic illnesses is growing rapidly, as they may provide a safer alternative to MSC treatments and have shown benefit in multiple preclinical animal models of injury and disease $(4,5)$.

EVs are membrane-bound spherical structures secreted from cells and ranging in size from $30-5,000 \mathrm{~nm}$ in diameter (6-9). They express similar plasma membrane proteins as their parent cells and contain biologically important cargo, including noncoding RNA, proteins, DNA, mitochondria, and lipids (6). EVs are important for cell-tocell communication and allow for the exchange of cellular components modulating downstream signaling pathways (6). The EV plasma surface components and internal EV contents all contribute to their unique function as signaling modulators, either via cargo delivery and/or direct activation of signaling pathways in recipient cells (6-9).
In preclinical studies, MSC-EVs have demonstrated reduced inflammation and improved organ function in multiple models. However, published studies have used a variety of different research designs which can make comparing the results of different studies difficult. Inconsistency in study design and reporting can complicate how to optimize future MSC-EV research, and limit identification of key features most important for efficacy in considering translational applications.

In a recent issue of Fournal of Extracellular Vesicles, Tieu and colleagues performed a thorough and highly useful meta-analysis of preclinical studies evaluating the effectiveness of MSC-EVs in models of acute and chronic respiratory diseases (10). Major parameters for study inclusion included use of in vivo animal models of respiratory injury and disease, and the administration of MSC-EVs which could be derived from any animal or tissue source. The authors screened 1,167 records identified from a MEDLINE/Embase search, with a final 52 articles included for data extraction. Additional analyses for risk bias and quality of reporting in study design were also performed on the selected articles. The final analysis focused on studies using models of acute lung injury (ALI), bronchopulmonary dysplasia (BPD), and pulmonary arterial hypertension $(\mathrm{PAH})$, which were the three most-studied conditions modeled in the studies included for data extraction.

The analyses clearly identified beneficial primary and secondary outcomes demonstrating efficacy of MSCEVs in ALI, BPD, and PAH in preclinical animal models. 
ALI in humans, resulting from various insults to the lung parenchyma or vasculature, has a significant burden of morbidity and mortality, as the world has become painfully aware during the COVID-19 pandemic, characterized by rapid onset and progression of respiratory failure (11-13). While the pathophysiology includes a severe inflammatory response resulting in damage to lung tissue, treatment with anti-inflammatory therapies has been mostly ineffective at improving outcomes in patients with ALI (14). This metaanalysis found MSC-EVs were effective in ALI models at improving the primary outcome of lung injury score and the secondary outcomes of survival, vascular permeability and BALF neutrophil count. BPD is a chronic lung disease in premature infants that is multifactorial in origin and remains a significant cause of morbidity and mortality (15). The lungs of infants with BPD have alveolar hypoplasia and decreased pulmonary angiogenesis, resulting in increased risk from subsequent pulmonary infection, inflammation, and injury throughout childhood. In BPD models MSCEVs were effective in improving the primary outcome of improved alveolarization and the secondary outcomes of lung permeability, BALF neutrophil count, and Fulton's index (a measure of right ventricular hypertrophy and an indicator of pulmonary hypertension). $\mathrm{PAH}$ is characterized by the remodeling of the pulmonary arteries, loss of vascular cross-sectional area, right ventricular hypertrophy, and pulmonary artery pressures of $>25 \mathrm{mmHg}$ leading to right heart failure and death $(16,17)$. There are limited treatment options available for PAH short of lung transplantation; current PAH treatments do not improve the abnormal pulmonary vascular remodeling or inflammation. The authors' analysis indicates that in PAH models, MSC-EVs were effective in reducing the primary outcome of right ventricular systolic pressure and the secondary outcome of Fulton's index. While the number of overall studies for each sub-group analysis was relatively small, this meta-analysis was able to show significant improvement of outcomes in all three modeled conditions after treatment with MSC-EVs, indicating that MSC-EVs have strong scientific promise for further research and development as a possible therapeutic avenue for respiratory diseases.

Tieu and colleagues went an important step further, comparing multiple important aspects of research design across the studies, including general study characteristics, animal model features, MSC and EV isolation and characterization techniques, MSC and EV modifications, and delivered dosage. The International Society for Cell and Gene Therapy (ISCT) and International Society for
Extracellular Vesicles (ISEV) have published guidelines on best laboratory and clinical practice for isolation and characterization of EVs and on the design and execution of research studies involving EVs $(18,19)$. The authors found that even with published guidelines on EV research, a high level of heterogeneity remained in how closely these guidelines have been followed in published research studies. Given the breadth of variables that are involved in generating MSC-EVs, subgroup analysis was performed to identify specific parameters across the studies which may be associated with enhancing $\mathrm{EV}$ efficacy. $\mathrm{EV}$ tissue source and route of administration to the animals (intravenous $v s$. intratracheal) were all equally effective across all three disorders. For EV isolation techniques ultracentrifugation appeared to have increased clinical benefit $v s$. tangential flow filtration (TFF) for both BPD and PAH (TFF was not used for EV isolation in the ALI studies). Finally, smaller EVs or a combination of small and large EVs were more efficacious than large EVs for ALI and BPD. EV size could not be evaluated in PAH since large EVs were not used in PAH studies. This effect of EV size seen in this analysis suggests that there may be a benefit for smaller EVs. Unfortunately, the overall sample sizes of these subgroup analyses were small; further studies looking at these questions are still needed.

Tieu and colleagues also assessed risk of bias and quality in preclinical study design using the SYRCLE tool, finding general inconsistency across the EV studies analyzed and making it impossible to have a clear report of bias and quality (20). They have called for better description and rigor of experimental parameters such as randomization, blinding, and sample size estimates to increase to robustness of the evidence for EV efficacy to better guide clinical translation.

A great deal of scientific research is aimed at finding new therapeutic strategies for pulmonary diseases such as ALI, BPD, and PAH, given the limited treatment options currently available. MSC-EVs have been shown to effective in the treatment of lung disease and injury in preclinical animal models $(4,5)$, however, there remain many basic scientific and clinical questions that need to be addressed before this technology can be translated to humans. Tieu and colleagues describe characteristics of MSC-EVs which appear important for increased efficacy in the treatment of respiratory disorders in preclinical animal models (10). This analysis strongly underscores the scientific premise, and promise, of using EVs for respiratory disease, and also begins to provide a highly useful framework which 
researchers can use to evaluate how their EV studies can be optimized for data collection and publication. This work underscores the importance of following the ISCT and ISEV guidelines for EV study design which would aid in study comparison and may help speed development and the rate of future successful EV research and translation $(18,19)$. This approach should be extended to other preclinical models in respiratory disease and beyond. This study also highlights other issues that have been previously raised, including the need for dose-response and biodistribution studies for EVs which will be critical for clinical translation. There needs to be head-to-head comparison of the different $\mathrm{EV}$ purification methodologies and of the different EV subtypes to enhance efficacy. Furthermore, guidelines on characterizing biomarkers and EV cargo important for cell signaling are needed.

This study provides important guideposts for ongoing research in the field; investigators can use these results when designing future studies; providing increased rigor and homogeneity across studies and allowing for meaningful cross-comparison of results. Increased consistency leading to more robust data should lead to consensus in the field regarding key characteristics of MSC$\mathrm{EV}$ therapies which can be translated into clinical trials. Although many scientific questions and technical issues remain to be resolved before MSC-EVs can make the transition from animal models to humans, these challenges can be overcome, yielding great promise for MSC-EVs as a potential therapeutic approach for a broad range pulmonary and other diseases.

\section{Acknowledgments}

Funding: None.

\section{Footnote}

Provenance and Peer Review: This article was commissioned by the editorial office, ExRNA. The article has undergone external peer review.

Conflicts of Interest: Both authors have completed the ICMJE uniform disclosure form (available at https://exrna. amegroups.com/article/view/10.21037/exrna-21-35/coif). The authors have no conflicts of interest to declare.

Ethical Statement: The authors are accountable for all aspects of the work in ensuring that questions related to the accuracy or integrity of any part of the work are appropriately investigated and resolved.

Open Access Statement: This is an Open Access article distributed in accordance with the Creative Commons Attribution-NonCommercial-NoDerivs 4.0 International License (CC BY-NC-ND 4.0), which permits the noncommercial replication and distribution of the article with the strict proviso that no changes or edits are made and the original work is properly cited (including links to both the formal publication through the relevant DOI and the license). See: https://creativecommons.org/licenses/by-nc-nd/4.0/.

\section{References}

1. Ntege EH, Sunami H, Shimizu Y. Advances in regenerative therapy: a review of the literature and future directions. Regen Ther 2020;14:136-53.

2. Pittenger MF, Discher DE, Péault BM, et al. Mesenchymal stem cell perspective: cell biology to clinical progress. NPJ Regen Med 2019;4:22.

3. Harrell CR, Jankovic MG, Fellabaum C, et al. Molecular mechanisms responsible for anti-inflammatory and immunosuppressive effects of mesenchymal stem cellderived factors. Adv Exp Med Biol 2019;1084:187-206.

4. Fujita Y, Kadota T, Araya J, et al. Clinical application of mesenchymal stem cell-derived extracellular vesicle-based therapeutics for inflammatory lung diseases. J Clin Med 2018;7:355.

5. Worthington EN, Hagood JS. Therapeutic use of extracellular vesicles for acute and chronic lung disease. Int J Mol Sci 2020;21:2318.

6. van Niel G, D'Angelo G, Raposo G. Shedding light on the cell biology of extracellular vesicles. Nat Rev Mol Cell Biol 2018;19:213-28.

7. Abels ER, Breakefield XO. Introduction to extracellular vesicles: biogenesis, RNA cargo selection, content, release, and uptake. Cell Mol Neurobiol 2016;36:301-12.

8. Mathieu M, Martin-Jaular L, Lavieu G, et al. Specificities of secretion and uptake of exosomes and other extracellular vesicles for cell-to-cell communication. Nat Cell Biol 2019;21:9-17.

9. Tkach M, Théry C. Communication by extracellular vesicles: where we are and where we need to go. Cell 2016;164:1226-32.

10. Tieu A, Hu K, Gnyra C, et al. Mesenchymal stromal cell extracellular vesicles as therapy for acute and chronic respiratory diseases: a meta-analysis. J Extracell Vesicles 
2021;10:e12141.

11. Thompson BT, Chambers RC, Liu KD. Acute respiratory distress syndrome. N Engl J Med 2017;377:562-72.

12. Sweeney RM, McAuley DF. Acute respiratory distress syndrome. Lancet 2016;388:2416-30.

13. Confalonieri M, Salton F, Fabiano F. Acute respiratory distress syndrome. Eur Respir Rev 2017;26:160116.

14. Fan E, Brodie D, Slutsky AS. Acute respiratory distress syndrome: advances in diagnosis and treatment. JAMA 2018;319:698-710.

15. Namba F. Mesenchymal stem cells for the prevention of bronchopulmonary dysplasia. Pediatr Int 2019;61:945-50.

16. Guazzi M, Naeije R. Pulmonary hypertension in heart failure: pathophysiology, pathobiology, and emerging clinical perspectives. J Am Coll Cardiol 2017;69:1718-34.

doi: 10.21037/exrna-21-35

Cite this article as: Worthington EN, Hagood JS. Metaanalysis brings rigor to preclinical studies of extracellular vesicles for respiratory disease. ExRNA 2022;4:9.
17. Galiè N, Humbert M, Vachiery JL, et al. 2015 ESC/ERS guidelines for the diagnosis and treatment of pulmonary hypertension. Rev Esp Cardiol (Engl Ed) 2016;69:177.

18. Théry C, Witwer KW, Aikawa E, et al. Minimal information for studies of extracellular vesicles 2018 (MISEV2018): a position statement of the International Society for Extracellular Vesicles and update of the MISEV2014 guidelines. J Extracell Vesicles 2018;7:1535750.

19. Dominici M, Le Blanc K, Mueller I, et al. Minimal criteria for defining multipotent mesenchymal stromal cells. The International Society for Cellular Therapy position statement. Cytotherapy 2006;8:315-7.

20. Hooijmans CR, Rovers MM, de Vries RB, et al. SYRCLE's risk of bias tool for animal studies. BMC Med Res Methodol 2014;14:43. 